\title{
Occlusion of the abdominal aorta during coronary angiography with fractional flow reserve due to migration of the left ventricle thrombus in a patient with thrombocytosis
}

\author{
Przemysław Lipski ${ }^{1}$, Jarosław Bugajski ${ }^{1}$, Magdalena Stachera ${ }^{2}$, Marek Piskozub ${ }^{3}$, Katarzyna Sznajder ${ }^{2}$, \\ Marek Gierlotka ${ }^{1}$ \\ ${ }^{1}$ Department of Cardiology, University Hospital in Opole, Faculty of Natural Sciences and Technology, University of Opole, Poland \\ 2Department of Radiology, University Hospital, Opole, Poland \\ ${ }^{3}$ Department of General and Vascular Surgery, University Hospital, Opole, Poland
}

Adv Interv Cardiol 2019; 15, 2 (56): 260-261 DOI: https://doi.org/10.5114/aic.2019.86021

A 56-year-old man, with a 5-year history of treated thrombocytosis, hypertension, anterior myocardial infarction (1998) and recent stroke, was admitted to our department with preliminary diagnosis of tumour of the heart. A homogeneous pedunculated tumour mass of $4.9 \times 1.7 \mathrm{~cm}$ attached to the apex of the left ventricle (LV) was revealed in transthoracic echocardiography (TTE) (Figure $1 \mathrm{~A}$ ). The TTE did not detect any significant regional wall motion abnormalities with an LV ejection fraction of $54 \%$. The patient was asymptomatic. The electrocardiogram revealed normal sinus rhythm without any signs of previous myocardial infarction. The Heart Team decided to perform coronary angiography (history of myocardial infarction) before surgical removal of the LV tumour mass (recent neurological ischemic event).

Coronary angiography was performed with a radial approach. Moderate stenosis in the mid-portion of the left anterior descending artery (LAD) was observed (Figure 1 B). Subsequently, physiological assessment of the lesion was performed with the pressure sensor located distal to the stenosis. The fractional flow reserve (FFR) was 0.81 (Figure $1 \mathrm{C}$ ). During the procedure the patient felt a sharp, progressive pain in his lower limbs. Imme- diately, TTE (Figure $1 \mathrm{D}$ ) and then a computed tomography angiography (CTA) scan (Figure $1 \mathrm{E}$ ) were performed, showing the migration of the LV mass and the complete occlusion of the abdominal aorta with embolic material (with moderate density and without contrast enhancement). The patient was immediately transferred for urgent surgery. Under general anaesthesia, the vascular surgeon using a Fogarty balloon catheter successful performed embolectomy through both femoral arteries. The whole embolic mass was removed and peripheral circulation restored. After the procedure the patient had only mild right lower limb paresis. The histopathological examination confirmed thrombotic nature of the mass. Cardiac magnetic resonance (CMR) detected only small, residual organized thrombus in the apex of the LV (Figure $1 \mathrm{~F}$ ) with mild wall motion abnormalities (lateral wall and apex). Furthermore, Lake Louise Consensus Criteria were positive for myocarditis with oedema and typical late gadolinium enhancement patterns in the middle and apical segments. Anticoagulation with a low-molecular-weight heparin was started until the postoperative wound healed over and after that continued with warfarin. The patient was discharged home in general good condition after 23 days. 

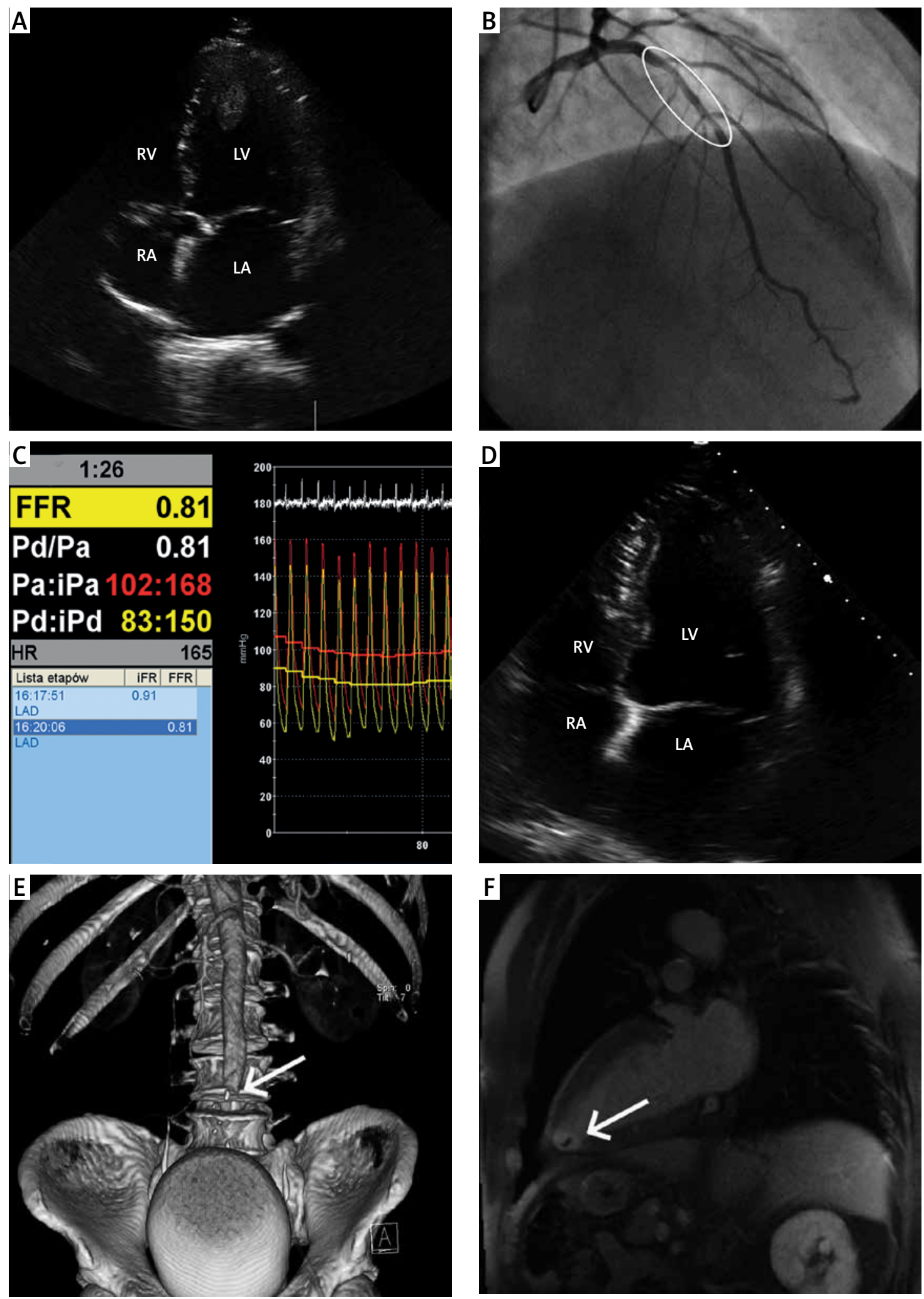

Figure 1. A - Transthoracic echocardiography in apical four-chamber view: the tumour mass in the apex of the left ventricle. B - Coronary angiography: Left anterior descending artery with intermediate lesion in the middle part (white ellipse). C - Fractional flow reserve (FFR). D - Transthoracic echocardiography in apical four-chamber view before vascular surgery: the tumour mass is not clearly visible in the apex of the left ventricle. E - Computed tomography angiography scan: 3D reconstruction of the occlusion of the abdominal aorta (white arrow). F - Cardiovascular magnetic resonance: remnants of thrombus in the apex of the left ventricle (white arrow) $L A$ - left atrium, $L V$ - left ventricle, $R V$ - right ventricle, $R A$ - right atrium. 